\title{
¿Es posible un intencionalismo pluralista?
}

\section{Is pluralistic intentionalism possible?}

\author{
ALICIA BERMEJO SALAR \\ Universidad de Murcia
}

Recibido:12/09/16 Aceptado: 2/12/16

\begin{abstract}
RESUMEN
En el debate actual entre las distintas teorías de la interpretación artística, uno de los principales argumentos que se aducen en contra del intencionalismo es su supuesta incompatibilidad con el pluralismo interpretativo, por ser una teoría monista. En este artículo discutiré, en primer lugar, el alcance de la propuesta de R. Stecker para hacer compatible el monismo y pluralismo y, en segundo lugar, la naturaleza del intencionalismo pluralista de G. Currie. Por último, intentaré mostrar qué tipo de pluralismo puede suscribir el intencionalismo sin necesidad de renunciar a su concepto de obra de arte y a su idea de interpretación artística.
\end{abstract}

PALABRAS CLAVE:

INTENCIONALISMO, PLURALISMO, INTERPRETACIÓN, INTENCIÓN, SIGNIFICADO.

\begin{abstract}
In contemporary discussions, one of the main arguments adduced against intentionalism is its supposed incompatibility with interpretative pluralism, due to its monist nature. In this paper I will discus the scope of R. Stecker's attempt to make monism and pluralism compatible and G. Currie's pluralist intentionalism. Finally, I will try to show what special kind of pluralism could be endorsed by intentionalism without needing to abandon its concept of art and its idea of artistic interpretation.
\end{abstract}

\section{KEYWORDS:}

INTENTIONALISM, PLURALISM, INTERPRETATION, INTENTION, MEANING 


\section{Intencionalismo, Monismo y PluRalismo}

En términos generales, podemos decir que el Intencionalismo es una teoría de la interpretación artística cuya tesis fundamental es que el significado de una obra de arte está determinado por la intención cumplida de su autor. Esta tesis implica un compromiso por parte del intencionalismo con dos ideas fundamentales. Por un lado, con una determinada concepción de lo que es una obra de arte, según la cual la obra es el producto de la acción intencional de su autor. Por otro lado, con una determinada concepción de la interpretación, según la cual su propósito fundamental es desentrañar el significado de la obra determinado por las intenciones cumplidas el autor. Estos dos compromisos implican, a su vez, que el intencionalismo vaya de la mano de una visión monista de la interpretación. Esta visión defiende que existe una única interpretación verdadera de las obras de arte, la cual, desde la perspectiva intencionalista se correspondería con el significado de la obra intencionalmente determinado por su autor. Por el contrario, desde un punto de vista pluralista, las interpretaciones posibles de una obra de arte no se agotan en lo que concierne al mero significado intencional de su autor, sino que existen múltiples interpretaciones aceptables. Es por ello que, generalmente, adoptar una visión pluralista de la interpretación ha implicado asumir, implícitamente, una forma de anti-intencionalismo.

Como vemos, las diferencias entre intencionalismo y pluralismo son de largo alcance, dado que tienen que ver con la noción misma de obra de arte y la visión de la interpretación que cada teoría mantiene. De hecho, una de las discusiones más importantes que se dan en el debate actual entre intencionalistas y anti-intencionalistas tiene que ver precisamente con la dicotomía entre una visión monista y una visión pluralista de la interpretación. Como ha señalado N. Carroll, (Carroll 2011, p. 181) el argumento fundamental que se aduce contra el intencionalismo, como teoría monista, es que limita la práctica interpretativa impidiendo que sea todo lo productiva que puede ser, ya que no admite como aceptable ninguna interpretación de la obra más allá de la correspondiente al significado intencional. Bajo este planteamiento, el intencionalismo sería una teoría de la interpretación deflacionaria al dejar fuera de su dominio ciertas interpretaciones posibles de la obra que podrían ser estética y artísticamente relevantes, incluso más relevantes que la propia interpretación intencional.

Por ésta y otras razones, ${ }^{1}$ el pluralismo ha sido generalmente concebido como un punto de equilibrio frente a los problemas que, por defecto o por exceso, presentan planteamientos interpretativos como el monismo y el relativismo. No

1 Las intuiciones a favor del pluralismo también obedecen a razones que van más allá de las dificultades que presenta el monismo. Estas razones tienen que ver con el hecho de que una actitud interpretativa pluralista está asociada tácitamente con una disposición hacia ciertos valores éticos, como la tolerancia, o con determinadas actitudes políticas, como la democracia. 
obstante, como vamos a ver a continuación, algunos intencionalistas han intentado o bien justificar una posible compatibilidad entre monismo y pluralismo, como es el caso de R. Stecker, o bien configurar un intencionalismo pluralista, como es el caso de G. Currie.

\section{LA COMPATIBILIDAD ENTRE MONISMO Y PLURALISMO SEGÚN STECKER}

Para responder a la cuestión de si monismo y pluralismo constituyen dos modelos interpretativos realmente incompatibles R. Stecker se dirige al núcleo de las disputas entre monistas y pluralistas: la discusión sobre la naturaleza de los enunciados de la interpretación. La polémica en torno al estatuto lógico de los enunciados interpretativos consiste en decidir si dichos enunciados tienen un carácter veritativo, es decir, si las interpretaciones de obras de arte son verdaderas o falsas, o no. Hablar de las interpretaciones de las obras de arte como siendo verdaderas o falsas es una fórmula recurrente en el lenguaje cotidiano, es parte de la forma en la que nos expresamos. Por ejemplo, habitualmente utilizamos expresiones del tipo «mucha gente cree que..., pero la verdadera interpretación es que...». Tomando un ejemplo de Wollheim (Wollheim 1997, p. 116), en el cuadro La instrucción paterna de G. Terborch, (1650) alguien podría considerar que la interpretación verdadera es que el cuadro representa a una joven recibiendo una pequeña reprimenda por parte de su padre, cuando la interpretación verdadera es que representa a una prostituta que pone precio a sus servicios.

Sin embargo, no todo el mundo considera que hablar de interpretaciones verdaderas sea un modo de expresión adecuado para referirse a obras de arte. J. Margolis ha defendido la tesis relativista para la interpretación considerando que podemos hablar de interpretaciones falsas, pero no de interpretaciones verdaderas en ningún sentido (Margolis 1987). Asimismo, otros planteamientos, como el anti-descriptivismo, se han basado en la idea de que en la medida en que no se pueda hablar de interpretaciones verdaderas tampoco tiene sentido hablar de interpretaciones falsas. ${ }^{2}$ Planteamientos como estos han propiciado el surgimiento de un criterio para calificar la naturaleza los enunciados de la interpretación alternativo al veritativo, a saber, el criterio de aceptabilidad. Este criterio también encuentra su justificación atendiendo a la manera en que nos expresamos, ya que es habitual decir que una interpretación es aceptable o inaceptable.

El criterio de aceptabilidad es el que rige en la interpretación desde el punto de vista del pluralismo, lo que significa que puede haber múltiples interpretaciones de una obra de arte, en tanto que los enunciados que constituyen dichas

No obstante, la motivación que rige en este trabajo es únicamente la de justificar si podemos ser intencionalistas y pluralistas en la interpretación de una obra de arte al mismo tiempo.

2 Cf. Stecker 1994, p. 198. 
interpretaciones carecen de valor de verdad, es decir, no son ni verdaderos ni falsos, sino aceptables o no aceptables. En este sentido, el criterio de aceptabilidad presenta diferencias considerables respecto al veritativo. Una diferencia consiste en que las nociones de verdad y falsedad, que rigen en el criterio veritativo, pertenecen al dominio la lógica bivalente, donde lo que se predica con verdad de una cosa, implica a su vez la falsedad de su contraria, mientras que la noción de aceptabilidad pertenece a un dominio multivalente, en el que aquello que se considera aceptable de una obra no implica que su contrario no pueda ser aceptable también. Otra diferencia es que el criterio veritativo no admite gradación, mientras que el criterio de aceptabilidad sí. Esto implica que no podemos decir que una interpretación es más o menos verdadera que otra, sino que una es verdadera y la otra no, mientras que sí podemos decir que una interpretación es más o menos aceptable que otra, siendo ambas aceptables.

Stecker ha defendido que verdad y aceptabilidad configuran dos criterios de clasificación de los enunciados interpretativos igualmente legítimos, pero que tienen que ser independientes. La razón es que no se pueden mezclar enunciados verdaderos y enunciados aceptables en una misma interpretación de una obra de arte. Esto es así porque, como hemos dicho, los enunciados interpretativos que se consideran verdaderos excluyen como falsos sus contrarios, de manera que la adicción de distintos juicios verdaderos, bajo una única interpretación global, nunca dará lugar a una interpretación de la obra que incluya enunciados contradictorios. Por el contrario, las interpretaciones basadas en enunciados que se rigen por el criterio de aceptabilidad no admiten la adición de distintos enunciados aceptables para constituir una interpretación general, ya que la aceptabilidad de un enunciado no implica la negación de la aceptabilidad del enunciado contrario. ${ }^{3}$

La distinción entre el criterio veritativo y el criterio de aceptabilidad entraña la clave de la estrategia de Stecker para desbloquear la oposición entre monismo y pluralismo (Stecker 1994). Para Stecker, monismo y pluralismo constituyen dos modelos de interpretación entre los que no podemos elegir, y entre los que no tenemos por qué elegir. Según el autor, el problema reside en pensar que la interpretación de obras de arte obedece a un propósito único y exclusivo. Los

3 En este sentido, Stecker ha proporcionado una clasificación adicional de las interpretaciones que se dan bajo uno y otro criterio, a saber, aquella que distingue entre interpretaciones comprensivas y no-comprensivas. Una interpretación comprensiva se constituye exclusivamente sobre la base de enunciados con carácter veritativo y es aquella que aglutina distintos enunciados interpretativos para dar lugar a una interpretación general que los reúne a todos. Por el contrario, las interpretaciones de naturaleza no-comprensiva están basadas en el criterio de aceptabilidad de los enunciados y en ella no es posible la adición de distintos enunciados interpretativos aceptables de una obra de arte bajo una misma interpretación global. Cf. Stecker 2003, p. 37 y Stecker 1997, p. 134. 
intérpretes pueden tener múltiples propósitos legítimos, ya sea desentrañar el significado intencional de la obra, tener una determinada experiencia, ampliar su capacidad imaginativa, adquirir un determinado conocimiento, etc. (Stecker 1994, p. 193). En este sentido, el autor defiende que la interpretación de la obra se regirá bajo el criterio veritativo o de aceptabilidad en función de cuál sea su propósito específico.

No obstante, el autor afirma que: «si una interpretación es aceptable o no varía de acuerdo con el propósito de aquellos que la producen o la reciben. Varía dependiendo de para qué es. [...] La corrección de una interpretación no varía en este sentido». ${ }^{4}$ Esto significa que un enunciado interpretativo es aceptable o no en función del propósito de la interpretación, mientras que un enunciado no será verdadero o no en función del propósito, sino que será verdadero en todo caso. Veamos un ejemplo para ver en qué sentido la aceptabilidad de un enunciado es relativa al propósito específico de la interpretación. Pongamos por caso la interpretación de la obra de Shakespeare Hamlet (1605) y pensemos en un enunciado interpretativo del tipo: «Hamlet padecía el complejo de Edipo». Para Stecker, esta interpretación de la obra sería aceptable si nuestro propósito interpretativo fuera, por ejemplo, ampliar nuestra experiencia de la misma interpretándola a la luz de una teoría específica como es el psicoanálisis.

Por el contrario, los enunciados interpretativos calificables como verdaderos no son relativos al propósito específico de la interpretación, es decir, las interpretaciones verdaderas lo son en términos absolutos. Uno de los propósitos interpretativos que implica enunciados de carácter veritativo es el de identificar el significado de la obra. Esto es así porque Stecker, como intencionalista, considera que cuando el propósito de la interpretación es desentrañar el significado hay que remitirse a la intención del autor; y la intención obedece al criterio veritativo, ya que es verdadero o falso que el autor intentó hacer tal o cual cosa. Por lo tanto, un enunciado del tipo «Shakespeare tenía la intención representar a Hamlet sintiendo un amor especial por su madre» sería verdadero con independencia del objetivo de la interpretación. Pero el enunciado interpretativo «Hamlet padecía el complejo de Edipo» no sería verdadero en una interpretación cuyo propósito fuera el significado. Esto es así porque el enunciado interpretativo que recoge la acción intencional del autor $-\ll$ Shakespeare tenía la intención de representar en Hamlet un caso del complejo de Edipo»- no es verdadero, dado que en esa

4 Stecker 1997, p. 193: «Whether an interpretation is acceptable varies according to the aim of those who produce or receive it. It varies depending on what it is for. [...] The correctness of an interpretation does not vary in this way». 
época todavía no se había desarrollado el psicoanálisis y, por lo tanto, Shakespeare no pudo haberse formado esa intención. ${ }^{5}$

Así pues, según Stecker, existen propósitos interpretativos que se adecuan mejor a la tesis monista y otros que se adecuan mejor a la pluralista. De esta manera, para Stecker, monismo y pluralismo tienen que ver con propósitos interpretativos diferentes que, a su vez, implican tipos de enunciados interpretativos diferentes. Por lo tanto, se podría hablar tanto de una interpretación verdadera y única de la obra bajo el paradigma monista, como de múltiples interpretaciones buenas y aceptables bajo el paradigma pluralista, de manera que monismo y pluralismo no serían incompatibles. Esta compatibilidad sería relevante en la medida en que si monismo y pluralismo fueran compatibles también deberían serlo el intencionalismo - como planteamiento que se considera, en principio, monista- y el pluralismo. Pero la compatibilidad entre monismo y pluralismo que resulta de la propuesta de Stecker no es realmente tal y, por lo tanto, no es coextensiva a la compatibilidad entre intencionalismo y pluralismo.

La particularidad del planteamiento de Stecker es que defiende un monismo para el significado de la obra y un pluralismo para la interpretación (Stecker 2003, p. 198). Es decir, considera que existe una interpretación verdadera que se corresponde con lo que el artista hizo en la obra intencionalmente. Pero, dado que los propósitos de la interpretación son diversos, aquellos que no sean relativos al significado sí admitirían el pluralismo, porque no exigirían restringirse a lo verdadero. Por el contrario, si el objetivo en la interpretación es el significado -objetivo de la interpretación para el cual es relevante la actitud interpretativa intencionalista- entonces hay que adoptar una actitud monista.

Ahora bien, la propuesta de Stecker, que tiene como objetivo hacer compatibles monismo y pluralismo, no se traduce en una compatibilidad entre intencionalismo y pluralismo. Stecker incluye las intuiciones a favor del pluralismo y su legitimidad como teoría interpretativa reconociendo la existencia de diversos objetivos interpretativos; objetivos para consecución de los cuales la intención del autor es irrelevante. Así pues, el planteamiento del autor no justifica una posible relación del pluralismo con el objetivo relevante para el intencionalismo, que es el del significado de la obra. Si esto es así, se podría cuestionar si monismo y pluralismo son compatibles en el sentido relevante, lo que significa que se pueda ser pluralista con el mismo objetivo interpretativo del intencionalismo, que es el significado. Sin embargo, en la propuesta de Stecker parece más bien que

5 En el punto IV defenderé que el intencionalismo entraña una actitud interpretativa compatible con pluralismo, según la cual un enunciado interpretativo «Hamlet padecía el complejo de Edipo» sí podría considerarse un enunciado interpretativo verdadero y compatible con una interpretación intencionalista de la obra. Para ello daré una idea de la relación que existe entre las distintas descripciones posibles de lo que un autor hace intencionalmente y los enunciados interpretativos que pueden llegar a formar parte de una interpretación verdadera de la obra. 
ambos paradigmas interpretativos encuentran su justificación en campos lógicos diferenciados, que también implican contextos interpretativos independientes. Si esto es así, la compatibilidad entre monismo y pluralismo resulta nimia, ya que es posible pensar que, en realidad, no son ni compatibles ni no compatibles, sino que no están relacionados en la interpretación en modo alguno. Por lo tanto, la propuesta no contribuye a la justificación de un intencionalismo pluralista, ya que no se traduce en la compatibilidad entre intencionalismo y pluralismo.

\section{El intencionalismo PluRalista De G. CURRIE}

Un autor que ha intentado mantener una visión intencionalista y pluralista de la interpretación es G. Currie. En su artículo «Interpretación y pragmática» (Currie 2012) el autor se adscribe a la tesis pluralista según la cual pueden existir dos o más interpretaciones posibles de una obra de arte sin que una sea mejor que las otras (Currie 2012, p. 186). Como ejemplo, Currie utiliza las dos interpretaciones que se pueden hacer de la historia de terror Otra vuelta de tuerca, escrita por Henry James (1898). Una de las dos interpretaciones posibles es que hay fantasmas que la institutriz ve; la otra es que la institutriz está loca y todo es producto de su imaginación. Según Currie, no hay nada en el texto que permita discernir cuál de las dos interpretaciones es más correcta (Currie 2012, p. 187) y, dado que James podría haber intentado representar tanto una cosa como la otra, considera que las dos interpretaciones son posibles y compatibles con la intención del autor. Por lo tanto, sería posible una interpretación de la obra intencionalista y múltiple, de modo que en este caso el intencionalismo sería compatible con el pluralismo.

Ahora bien, Currie también acepta la idea de que la intención del James podría haber sido escribir una obra ambigua. En este sentido, afirma que «a menudo vemos la ambigüedad como un fracaso de la comunicación, pero puede ser intencionada, y puede ser comunicada de tal modo que la advirtamos porque veamos que era intencionada» (Currie 2012, p. 188). Currie parece considerar que éste era precisamente el caso de Otra vuelta de Tuerca, ya que afirma que «hay razones para pensar que Henry James buscaba ese tipo de ambigüedad en su relato» (Currie 2012, p. 188). Si esto es así, la intención del autor era hacer una obra con significado ambiguo y, si esa ambigüedad es identificable en su obra-como parece que lo es-podemos decir que el autor cumplió su intención. Así pues, a la hora de interpretar Otra vuelta de tuerca tendríamos dos opciones:

(i) Opción interpretativa intencionalista pluralista: que la obra tenga dos interpretaciones aceptables, que se corresponden con las dos lecturas opuestas que son compatibles con las intenciones posibles del autor que refleja el texto, a saber, que la institutriz esté loca y que la institutriz esté cuerda.

(ii) Opción interpretativa intencionalista monista: que la obra tenga una única interpretación verdadera, que se corresponde con la interpretación de la 
obra como siendo ambigua; interpretación acorde con las intenciones cumplidas de su autor.

Sobre la opción (i) podríamos preguntarnos si un texto puede ser evidencia de dos cosas opuestas al mismo tiempo o si lo que ocurre es que el texto evidencia una sola cosa: que la institutriz está ambiguamente loca o cuerda. Para Currie «si la lectura más cercana, más delicada, del relato de James no aclara si está loca o no, entonces ella misma no está ni loca ni no-loca» (Currie 2012, p. 190). Pero, si esto es así, sería pensable que la obra no tuviera dos interpretaciones posibles (i), como sostiene Currie, sino sólo una, a saber, aquella que entiende la obra como siendo ambigua, es decir, sólo la opción (ii). Además, dado que para el intencionalismo la interpretación verdadera de la obra se corresponde con las intenciones cumplidas del autor, un intencionalista se inclinaría por la opción (ii). Sin embargo, a pesar de saber cuál era la intención de James y que fue cumplida, ¿por qué Currie no considera que solamente la interpretación (ii) es la verdadera e insiste en hablar de dos interpretaciones posibles (i)?

Podríamos decir que es porque Currie no cree que «haya una idea coherente de la realidad en el mundo de las obras que haga verdadera una interpretación» (Currie 2012, p. 189). Sin embargo, afirmar que existe una interpretación verdadera de la obra no significa que tengamos que decidir entre mantener que la institutriz está loca o que no está loca, porque interpretando la obra según las intenciones cumplidas de James, no es ni una cosa ni la otra. El autor no intentó que la institutriz estuviera loca, pero tampoco intentó que fuera cuerda, así como tampoco intentó que fuera las dos cosas al mismo tiempo; cosa que sería imposible. Lo que intentó es otra cosa distinta, a saber, que fuera ambigua entre la locura y la cordura, es decir, que no fuera claramente ninguna de las dos cosas. Y si esto es lo que el autor intentó y cumplió, entonces no es posible interpretar la obra de forma pluralista e intencionalista, porque no tenemos dos interpretaciones, sino una sola: aquella según la cual la institutriz es ambiguamente loca o ambiguamente cuerda. Esto no permite a hablar de dos interpretaciones aceptables, ya que «ser ambiguamente loca» y «ser ambiguamente cuerda» es lo mismo. Si se mantiene la opción interpretativa pluralista-opción (i)-, es a costa de sacrificar la verdadera interpretación intencionalista -opción interpretativa (ii).

Asimismo, podríamos preguntarnos si es acaso deseable poder interpretar la obra de manera pluralista e intencionalista, ya que el principio de justificación del pluralismo que asume Currie, en virtud del cual tener «varias cosas buenas es mejor que tener una» (Currie 2012, p. 193) podría ser discutible. ¿Realmente es mejor tener dos interpretaciones buenas de la obra que tener sólo una, si ésta es mejor que las otras? Lo mejor de Otra vuelta de tuerca no es que podamos leerla un día creyendo que la institutriz está loca y que al día siguiente podamos leerla creyendo que no está loca -lo que sería tener dos cosas buenas-, sino que 
podamos, en la misma lectura y en la misma interpretación, ver a la institutriz como no siendo claramente loca o cuerda. Es cierto que uno puede leer la obra día pensando una cosa y otro día pensando la otra. Pero eso no es óbice para que también sea posible que uno lea la obra debatiéndose entre una posibilidad o la otra, es decir, leer la obra dudando, oscilando entre explicar la actuación de la institutriz como la propia de una persona cuerda o como la propia de una persona demente. Es pensable que lo bueno de la obra no es que admita dos interpretaciones distintas, sino que admite una única interpretación que es mejor que las dos. Así pues, el principio pluralista de que tener varias cosas buenas es mejor que tener sólo una no se cumple necesariamente.

Siguiendo la nomenclatura de Currie, diríamos que las dos interpretaciones posibles de Otra vuelta de tuerca son «óptimas» (Currie 2012, p. 189), pero la interpretación que no las divide es la mejor. Podemos considerar que las dos posibles lecturas del texto dan lugar a dos interpretaciones distintas, como hace Currie, o podemos considerar que las dos lecturas posibles del texto se pueden integrar en la misma interpretación, concibiéndola como ambigua entre las dos posibilidades. Por lo tanto, desde un punto de vista intencionalista, no es evidente que podamos interpretar Otra vuelta de tuerca de forma pluralista, o al menos no parece evidente que esa interpretación sea mejor artística o estéticamente que la interpretación monista acorde con la intención del autor. No obstante, ¿significa esto que los intencionalistas no pueden ser pluralistas en ningún sentido?

\section{UN PLURALISMO 'RESTRINGIDO’}

Por último, recogiendo los problemas fundamentales de los planteamientos de Stecker y Currie, intentaré esbozar la clase de pluralismo que creo que es compatible con la visión intencionalista de la interpretación. Una manera de comprender cómo se pueden relacionar intencionalismo y pluralismo en el sentido relevante es comprender cómo está comprometido el intencionalismo con la idea de interpretación verdadera. Como he dicho, para el intencionalismo, existe una única interpretación verdadera de la obra, aquella que desvela el significado determinado por las intenciones cumplidas de su autor. Según esta tesis, la naturaleza del intencionalismo sería manifiestamente monista. Sin embargo, también es cierto que, en ocasiones, el intencionalismo tiene que mantener una actitud interpretativa pluralista - que no un pluralismo en sentido estricto-, ya que es evidente que no siempre se puede establecer cuál es la interpretación de la obra acorde con las intenciones cumplidas de su autor. Ésa actitud pluralista, que subyace en la visión intencionalista de la interpretación, es lo que denominaré pluralismo restringido. Veamos en qué consiste.

A diferencia de lo que ocurría en el caso de Otra vuelta de tuerca, muchas veces no sabemos cuál era la intención del autor, ni si quedó manifiesta en la obra. En estos casos, el intencionalismo opera con múltiples interpretaciones 
de la obra; todas ellas compatibles con las posibles intenciones del autor. Estas interpretaciones posibles son interpretaciones aceptables desde el punto de vista intencionalista en la medida en que sean potencialmente verdaderas. La diferencia es que para el pluralismo, digamos, no-restringido, una interpretación no necesita ser potencialmente verdadera para ser aceptable.

Esta diferencia se puede ilustrar tomando un ejemplo de N. Carroll. Generalmente se considera que la película de Edward Wood Plan 9 de espacio exterior (1956) es una de las peores películas filmadas (Carroll 2001, p. 174). La intención del director era hacer una película bajo los cánones de Hollywood, pero el resultado -ya fuera por la falta de presupuesto, por su falta de talento, o por una combinación de ambas-fue desastroso para cumplir con esa intención. Para un pluralista, además de la interpretación según la cual Plan 9 del espacio exterior es una obra fallida, también sería una interpretación aceptable aquella en la que podemos ver la película como una obra de vanguardia que, en lugar de intentar seguir un determinado código cinematográfico, lo subvierte y parodia. Así pues, aunque la interpretación de la obra en la que se concibe como una transgresión de los cánones de Hollywood no puede ser correctamente conectada con la intención de su autor, podría ser una interpretación aceptable desde el punto de vista del pluralismo. Pero además podría ser una interpretación más satisfactoria, ya que puede hacer a la película más relevante desde el punto de vista estético y artístico.

Por el contrario, para un intencionalista interpretar Plan 9 del espacio exterior como una transgresión de los cánones del cine de Hollywood no es aceptable porque no es compatible con lo que su autor hizo intencionalmente: el autor no intentó transgredir esas reglas, sino seguirlas. Para el intencionalismo, los enunciados interpretativos aceptables no constituyen un tipo absolutamente distinto de los verdaderos, en la medida en que sólo son aceptables aquellos que podrían llegar a ser verdaderos. Unos lo serán y otros no, pero la condición para que sean aceptables es que puedan llegar a ser verdaderos. Los que no puedan ser verdaderos porque contravienen lo que podría haber intentado el autor tampoco serán aceptables. Es precisamente ahí donde reside la diferencia fundamental entre intencionalismo y pluralismo.

Estas interpretaciones aceptables para el intencionalismo tienen carácter provisional, ya que cuentan como tales sólo hasta el momento de confirmarse la intención del autor, aunque en muchos casos esto no sea posible, ya que frecuentemente nos encontramos con múltiples hipótesis compatibles con lo que el autor podría haber intentado. Este carácter provisional hace que las interpretaciones aceptables queden mejor caracterizadas si nos referimos a ellas como «hipótesis interpretativas». Como hemos visto, las hipótesis sobre el significado de la obra que contempla el intencionalismo sólo son aceptables si son coherentes con lo que sea aceptable pensar que fue la intención del autor. Es decir, para interpretar 
una obra de manera intencionalista hacemos hipótesis que se perfilan en función de lo que podemos suponer que el autor podría haber intentado. De esta manera, la intención funciona como criterio para decidir sobre la aceptabilidad de una hipótesis interpretativa, considerando que es aceptable si y sólo si puede llegar a ser confirmada como verdadera; entendiendo por verdadera aquella que corresponde con las intenciones cumplidas del autor. Así pues, cabe concluir que la relación del intencionalismo con la idea de interpretación verdadera existe y, por ello, es una teoría monista. Pero está condicionada por las dificultades para confirmar o refutar las hipótesis interpretativas aceptables, lo que le lleva a funcionar en la práctica con el criterio de aceptabilidad de los enunciados en el dominio de lo que he denominado un pluralismo restringido.

Esta visión de la interpretación como un proceso formulación de hipótesis sobre el significado de la obra nos permite establecer una relación entre los criterios veritativo y de aceptabilidad, lo que permite, a su vez, vincular intencionalismo y pluralismo. El intencionalismo no es absolutamente incompatible con el pluralismo porque, a diferencia de lo que sostenía Stecker, el criterio de verdad y el criterio de aceptabilidad sí pueden coincidir en la interpretación aun cuando su propósito sea desentrañar el significado de la obra, pero en distintas fases de la interpretación. Veamos por qué.

El intencionalismo no tiene por qué suscribir la brecha que tradicionalmente se ha establecido entre la verdad y la aceptabilidad de los enunciados, ya que cuando hacemos una hipótesis no nos encontramos exclusivamente en el dominio de la verdad. Esto es así porque una hipótesis verdadera no es ya una hipótesis, las hipótesis tienen que ser, por su propia definición, aceptables, no verdaderas. Esta idea minimiza la dicotomía entre el criterio veritativo y el criterio de aceptabilidad, ya que podemos considerar que una hipótesis es un enunciado aceptable susceptible de confirmarse como verdadero. Así pues, podemos decir que el intencionalismo es pluralista en cierto grado, porque lo es en una determinada fase de la interpretación, a saber, aquella en la que no existe un criterio para decidir cuál de las múltiples hipótesis aceptables sobre la intención del autor es la verdadera. Es en este sentido en el que podemos decir que los intencionalistas manejan un pluralismo restringido; restringido porque no todos los enunciados interpretativos pueden llegar a ser hipótesis aceptables, pero pluralista si no existe una única hipótesis interpretativa posible. De esta manera, vemos que en la interpretación se pueden manejar tres tipos de interpretaciones:

a. Interpretaciones verdaderas

b. Interpretaciones aceptables potencialmente verdaderas

c. Interpretaciones aceptables no-potencialmente verdaderas 
Bajo los postulados del pluralismo restringido que propongo, el intencionalismo sólo manejaría en la interpretación los tipos a y b, mientras que el pluralismo los tendría en cuenta todos, o el b y el c, o sólo el c, según el caso.

Llegados a este punto cabe preguntarse qué clase de hipótesis son aceptables para el intencionalismo, es decir, cuáles son esas hipótesis potencialmente verdaderas con las que trabaja el pluralismo restringido del intencionalismo. La idea de que existe una interpretación verdadera de una obra de arte es ciertamente problemática. Sin embargo, un conjunto de enunciados verdaderos sobre la obra podría proporcionarnos, al menos, una aproximación a lo que sería su interpretación verdadera. Este conjunto no se compone exclusivamente de los enunciados que afirman meras trivialidades sobre la obra, sino que incluye enunciados que permiten una visión de la obra más interesante desde el punto de vista interpretativo, sin ir en detrimento de la intención del autor. Esta cuestión nos lleva directamente al origen del debate entre intencionalistas y pluralistas que venía de la objeción que afirma que el intencionalismo es una teoría de la interpretación demasiado restrictiva. El pluralismo restringido del intencionalismo que he defendido contribuye a la solución de este problema, ya que muestra que si bien la capacidad del intencionalismo como teoría interpretativa es más restringida que la del pluralismo, también es cierto que es más amplia que la del mero monismo que se le atribuye tradicionalmente. Veamos por qué con un ejemplo.

En la obra de Shakespeare El Rey Lear (1605), tan verdadero es el enunciado «el rey Lear era el padre de Cordelia», como el enunciado «el rey Lear tenía dudas sobre el amor que su hija Cordelia sentía por él». La verdad de un enunciado como el primero resulta trivial desde el punto de vista interpretativo, pero la verdad del segundo no es trivial porque nos permite añadir al conjunto de enunciados interpretativos otros más interesantes. Es decir, que se pueda afirmar con verdad que el rey Lear dudaba del amor de Cordelia da visos de aceptabilidad a hipótesis interpretativas más complejas que, en tanto que son potencialmente verdaderas, son también aceptables. Por ejemplo, la interpretación en clave epistemológica que ha hecho S. Cavell (Cavell 2003). El autor considera que El rey Lear constituye una instancia del problema de las otras mentes atendiendo al hecho de que su protagonista duda de los sentimientos de su hija, dada su incapacidad de tener un conocimiento certero sobre los estados mentales ajenos. Si es posible pensar que Shakespeare tenía la intención representar al rey Lear como siendo escéptico sobre los sentimientos de su hija, entonces la interpretación de Cavell sería una hipótesis interpretativa aceptable porque sería potencialmente verdadera. ¿Pero, cómo podemos saber si esta interpretación es verdadera y no sólo potencialmente verdadera? El criterio que propongo es el siguiente: toda descripción posible de la acción intencional del autor constituye una hipótesis interpretativa aceptable, que formaría parte de 
la interpretación verdadera de la obra si fuera una descripción correcta, y no meramente posible, de dicha acción intencional.

Supongamos que la interpretación de Cavell se puede formular mediante el siguiente enunciado: «La obra de Shakespeare El rey Lear representa un caso del problema de las otras mentes». En principio, se podría considerar que esta interpretación no puede ser verdadera, desde el punto de vista del intencionalismo, en la medida en que resulta anacrónico pensar que Shakespeare pudiera haber tenido la intención de representar este problema epistemológico. ${ }^{6}$ Esto implica que el enunciado «Shakespeare tenía la intención de representar el problema de las otras mentes en su obra El rey Lear» sería falso. Pero, bajo el criterio para decidir sobre la verdad de las hipóstesis que he formulado, resultaría que la hipótesis interpretativa de Cavell podría considerarse verdadera si se correspondiera con una descripción correcta de la acción intencional del autor. Por ejemplo, el enunciado «Shakespeare tenía la intención de representar al rey Lear dudando de los sentimientos de Cordelia» es una de las posibles descripciones de la acción intencional del autor. Es por ello que la interpretación de Cavell sí se podría considerar verdadera, ya que se sostiene sobre una descripción correcta de la acción intencional del autor. Por lo tanto, la obra representa un caso del problema de las otras mentes, ya que aunque Shakespeare no supiera en qué consiste este problema, lo ejemplifica en su obra al representar al rey Lear dudando de los estados mentales de su hija. Se trata de una interpretación acorde con la intención cumplida del autor que queda de manifiesto en la obra. Por el contrario, interpretar Plan 9 del espacio exterior como una obra transgresora no es compatible con una descripción correcta de lo que Wood hizo intencionalmente, que fue una mala imitación de los cánones de Hollywood. No se trata en este caso de una interpretación de la película más compleja, basada en una descripción correcta de la acción intencional del autor, sino de una interpretación basada en una descripción errónea y falsa, por lo tanto, sería una interpretación inaceptable.

Como vemos, las descripciones posibles de la acción intencional del autor son el soporte para la formulación de hipótesis interpretativas aceptables. Es decir, constituyen el criterio para determinar cuáles pueden ser estas hipótesis en el marco de un pluralismo restringido. De esta manera, el intencionalismo

6 Aunque tiene su orígenes en la epistemología cartesiana, el problema de las otras mentes no fue consolidado como problema filosófico hasta el siglo XIX. $C f$. http://plato.stanford.edu/ entries/other-minds/\#1

7 Este mismo argumento se aplicaría también al ejemplo que he utilizado en el apartado II. Se puede interpretar Hamlet como un caso del complejo de Edipo desde un punto de vista intencionalista de la interpretación, ya que esta interpretación está justificada por una descripción verdadera de la intención del autor, a saber, que Shakespeare tenía la intención de representar a Hamlet sintiendo un amor especial por su madre y cumplió su intención en la obra. 
puede acoplar a la interpretación verdadera de una obra muchas hipótesis interpretativas que se corresponden con descripciones más complejas, agudas, brillantes, lúcidas, de la acción intencional y que, por lo tanto resultan estética y artísticamente más relevantes en la interpretación. Es por ello que el intencionalismo no tiene que comprometerse necesariamente con el pluralismo para poder abarcar en la interpretación intencionalista de una obra todos los múltiples enunciados interpretativos que considere bien dirigidos. En última instancia, resulta que si bien es cierto que el intencionalismo mantiene meramente una actitud pluralista en la interpertación -que no un pluralismo en sentido estricto-, también es cierto que se puede cuestionar que el intencionalismo tenga que ir más allá de lo que sería un pluralismo restringido, en aras de evitar la objeción de que es una teoría interpretativamente deflacionaria. Que el intencionalismo sea monista no significa que sea reduccionista en la interpretación porque la interpretación intencionalista de la obra contiene distintas capas de complejidad relativas a la complejidad de las descripciones de la acción intencional del autor que estemos justificados a manejar.

\section{CONCLUSIÓN}

El pluralismo restringido no es un tipo de pluralismo formulado ad hoc para encajarlo con los principios del intencionalismo, ya que no pretende ser una teoría interpretativa, sino una descripción de la actitud interpretativa propia de los que interpretan desde el punto de vista intencionalista. Esta descripción proporciona una aproximación de sentido común a lo que hacemos al interpretar: barajamos varias posibilidades mientras no conocemos la más certera. Cuando contamos con un conocimiento que nos permite discernir cuál es la interpretación más adecuada, el resto de posibilidades pierden su relevancia como interpretaciones de la obra. Eso no significa que carezcan de todo interés, sino que ya no las consideraremos como alternativas interpretativas. Esto muestra que la interpretación intencionalista sólo incluye el pluralismo de una forma muy sutil, ya que el intencionalismo no maneja diversas interpretaciones posibles porque se comprometa con la idea de que la obra puede tener múltiples interpretaciones posibles, sino porque no hemos alcanzado la verdadera. No obstante, hablar de interpretación verdadera no tiene por qué implicar que una obra está cerrada desde el punto de vista interpretativo, porque, como hemos visto, se pueden formular muchas descripciones correctas de la acción intencional que den soporte a hipótesis interpretativas aceptables que se sumen la interpretación verdadera de la obra. De esta manera, es evidente que la motivación de los intencionalistas para ser pluralistas difiere con mucho de la motivación general de los pluralistas. No podría ser de otra manera cuando ambas teorías parten de una concepción diferente de lo que es una obra de arte y de cuáles son los objetivos a la hora de interpretar. En definitiva, esto indica que el intento de 
relacionar la visión intencionalista y la visión pluralista de la interpretación en términos de compatibilidad podría estar condenado al fracaso. Pero el hecho de que intencionalismo y pluralismo no sean compatibles tampoco significa que no puedan estar relacionados en modo alguno. Dando respuesta a la pregunta sobre si es posible un intencionalismo pluralista, que da título a este trabajo, me inclinaría por decir que en sentido estricto la respuesta es no, pero en sentido restringido la respuesta es sí.

\section{REFERENCIAS BIBLIOGRÁFICAS}

CARROLL, N. (2001): Beyond Aesthetics. Philosophical Essays. Cambridge: Cambridge University Press.

CAVELL, S. (2003): Disowning Knowledge in Seven Plays of Shakespeare. Cambridge: Cambridge University Press.

CURRIE, G. (2012): «Interpretación y pragmática», Artes \& Mentes. Madrid: Antonio Machado.

MARGOLIS, J. (1987): «Robust Relativism», en Margolis, J., (ed.) Philosophy Looks at the Arts. Contemporary Readings in Aesthetics. Philadelphia: Temple University Press.

STECKER, R. (1994): «Art Interpretation», The Journal of Aesthetics and Art Criticism, vol. $52, \mathrm{n}^{\circ} 2$, Spring.

STECKER, R. (1997): «Critical Monism and Critical Pluralism: How to Have Both», Artworks. Definition, Meaning and Value. Pennsylvania: The Pennsylvania State University Press.

- - , (2003): Interpretation and Construction. Art, Speech, and the Law. Oxford: Blackwell Publishing.

WOLLHEIM, R. (1997): La pintura como arte. Madrid: Visor. La balsa de la medusa.

Alicia Bermejo Salar es doctora en Filosofía por la Universidad de Murcia

Líneas de Investigación:

Estética y Filosofía del Lenguaje

Publicaciones recientes:

"Is Moderate Intentionalism Necessary?", en Proceedings of the Conference 2014 de la European Society for Aesthetics, http://www.eurosa.org/volume-6/ 
"The Analogy between Arstistic and Linguistic Meaning: The Linguistic Model of Intentionalism Revisited" en Proceedings of the Conference 2013 de la European Society for Aesthetics, http://www.eurosa.org/volume-5/

Correo electrónico: aliciabs@gmail.com 